\title{
A NOTE ON OPTIMAL EXPECTED UTILITY OF DIVIDEND PAYMENTS WITH PROPORTIONAL REINSURANCE
}

\author{
XIAOQING LIANG AND ZBIGNIEW PALMOWSKI
}

\begin{abstract}
In this paper, we consider the problem of maximizing the expected discounted utility of dividend payments for an insurance company that controls risk exposure by purchasing proportional reinsurance. We assume the preference of the insurer is of CRRA form. By solving the corresponding Hamilton-Jacobi-Bellman equation, we identify the value function and the corresponding optimal strategy. We also analyze the asymptotic behavior of the value function for large initial reserves. Finally, we provide some numerical examples to illustrate the results and analyze the sensitivity of the parameters.
\end{abstract}

KEYWORDS. Stochastic optimal control $\star$ Hamilton-Jacobi-Bellman equation $\star$ Optimal dividend $\star$ Proportional reinsurance

\section{CONTENTS}

1. Introduction

2. HJB equation

3. Non-cheap reinsurance

4. Cheap reinsurance

5. Numerical examples

6. Conclusions

References

Date: September 12, 2018.

This work is partially supported by the National Science Centre under the grant 2015/17/B/ST1/01102.The first author is partially supported by the National Natural Science Foundation of China (11571189) and High School National Science Foundation of Hebei Province (QN2016176). Both of the authors kindly acknowledge partial support by the project RARE -318984, a Marie Curie IRSES Fellowship within the 7th European Community Framework Programme. 


\section{INTRODUCTION}

In recent years there has been increasing attention towards the utilization of stochastic control theory to insurance-related problems. This is due to the fact that a company, such as a property-liability insurance company or a pension-fund management company, can control reinsurance strategies or investment strategies and can pay dividends to maximize (or minimize) a certain objective function under different constraints. Two kinds of risk processes have been considered. The first one concerns classical CramérLundberg process being drift process minus compound Poisson process, see e.g. Buhlmann [9], Hipp and Plum [17], Azcue and Muler [6, 7]. Later this case was generalized to the spectrally negative Lévy risk process, see Avram et al. [4, 5], Kyprianou and Palmowski [23], Loeffen [25, 27], Loeffen [26] and references therein. The second risk process, considered also in this paper, is a diffusion surplus risk model. In this model, the liquid asset processes of the corporation are driven by Brownian motion with constant drift and diffusion coefficients. The drift term corresponds to the expected profit per unit time, while the diffusion term is interpreted as risk. The classic studies on this subject are those by Gerber and Shiu [16], Jeanblanc and Shiryaev [21], Cadenillas et al. [11], Asmussen and Taksar [3], Asmussen et al. [2], Bai and Guo [8], Højgaard and Taksar [18, 19], Paulsen [30, 31], Zhou [34], David Promislow and Young [14] and many others. The details can be found in the survey paper Albrecher and Thonhauser [1] and in the book Schmidli [33].

The goal of this paper is to maximize the expected discounted utility of dividend payments for an insurance company whose reserve evolves in time according to a diffusion process and which controls risk exposure by purchasing proportional reinsurance. That is, in this paper we formally consider the following optimization problem.

Let $(\Omega, \mathcal{F}, P)$ denote a complete probability space endowed with information filtration $\left\{\mathcal{F}_{t}\right\}_{t \geq 0}$ and $\left\{B_{t}\right\}_{t \geq 0}$ be a standard Brownian motion adapted to the filtration. Let $R$ be a risk process being a Brownian motion with drift:

$$
R_{t}=(1+\theta) a t-Y_{t}, \quad R_{0}=x
$$

for the aggregate cumulative amount of claims counted up to time $t$ :

$$
d Y_{t}=a d t-b d B_{t}, \quad Y_{0}=0,
$$

where $a$ and $b$ are positive constants, $x \geq 0$ is the initial surplus, $(1+\theta) a$ is the premium rate with the safety loading $\theta>0$.

Apart of the risk process $R_{t}$ we will consider the dividend payments. Let $C=\left(C_{t}\right)_{t \geq 0}$ be an adapted and nondecreasing process representing all accumulated dividend payments up to time $t$. In our model we assume that $\left(C_{t}\right)_{t \geq 0}$ is absolutely continuous with respect to Lebesgue measure. Hence we suppose that the process $C$ admits almost surely a density process denoted by $c_{t} \geq 0$ modeling the intensity of the dividend payments in continuous time.

In our model, we add another new and very important feature in the context of dividend payments with utility function. We consider reinsurance policy, in which part of the premium rate $(1+\eta) q_{t} a$ for some proportion $q_{t} \in[0,1]$ is diverted to some reinsurer who will cover $q_{t}$ of arrived claims $Y_{t}$. In this way, the insurance company can reduce its risk exposure and therefore the reinsurance has been extensively studied, see for example Asmussen et al. [2], Azcue and Muler [6], Choulli et al. [12], Chen et al. [10], Højgaard and Taksar [18, 19], Liang and Young [24], Zhou and Yuen [32], and references therein.

Thus the reserve process $X_{t}^{\pi}$ evolves as follows:

$$
d X_{t}^{\pi}=\left((1+\theta) a-\left(1-q_{t}\right) a\right) d t+b\left(1-q_{t}\right) d B_{t}-(1+\eta) q_{t} a d t-c_{t} d t
$$




$$
=\left(\theta-\eta q_{t}\right) a d t+b\left(1-q_{t}\right) d B_{t}-c_{t} d t
$$

where $\pi$ in the superscript denotes a strategy which is described by a two-dimensional stochastic process $\left(q_{t}, c_{t}\right)$ that supposed to be chosen in optimal way, where the criterium for the optimality will be specified later. We will assume that $\eta \geq \theta$. When $\eta>\theta$, the fraction of the premiums diverted to the reinsurer is larger than that of each claim covered by the reinsurer, this is called non-cheap reinsurance. When $\eta=\theta$, we say the reinsurance is cheap. Both of these cases will be considered in this paper.

We observe the regulated process $X_{t}$ until the time of ruin:

$$
\tau=\inf \left\{t \geq 0: X_{t}^{\pi}<0\right\} .
$$

We define the target value function as

$$
V(x)=\max _{\pi \in \Pi} \mathbb{E}_{x}\left[\int_{0}^{\tau} e^{-\beta s} u\left(c_{s}\right) d s\right],
$$

where $\beta(>0)$ is a discount factor, $u$ is some fixed utility function, $\mathbb{E}_{x}$ means expectation with respect to $\mathbb{P}_{x}(\cdot)=P\left(\cdot \mid X_{0}=x\right)$ and maximum is taken over all admissible strategies $\Pi$. A strategy $\pi$ is said to be admissible if $\left(q_{t}, c_{t}\right)$ is $\mathcal{F}_{t}$-progressively measurable and satisfies $0 \leq q_{t} \leq 1, c_{t} \geq 0$ for all $t \geq 0$. Finally, we assume that the ruin cannot be caused by the dividend payment. Usually it is assumed that $u: \mathbb{R}_{\geq 0} \rightarrow \mathbb{R}_{\geq 0}$ is differentiable and nonnegative.

In fact, in this paper we will consider only the Constant Relative Risk Aversion (CRRA) utility function:

$$
u(c)=\frac{c^{p}}{p}, \quad p \in(0,1) .
$$

For above dividend problem we will prove the verification theorem producing the Hamilton-JacobiBellman (HJB) equation for optimal value function. This is done in Section_ Later (under some additional technical assumptions) we will solve it producing optimal strategy which appear to be a Markovian one, that is $q_{t}=q\left(X_{t}^{\pi}\right)$ and $c_{t}=c\left(X_{t}^{\pi}\right)$ for some functions $q$ and $c$ given explicit. In particular, for utility function (1.4) we will prove that when the reserve is sufficiently small (less than identified level $x^{*}$ ), insurance company is willing to buy part of reinsurance as well as diverting part of premium. When the reserve is larger than $x^{*}$ then the insurance company is able to afford all arrived claims and the optimal strategy excludes the purchase of reinsurance. Following Hubalek and Schachermayer [20] we also identify the optimal strategy for large reserves as $x \rightarrow \infty$. Both separate cases of non-cheap and cheap reinsurance polices are considered in Sections 3 and 4 . We also present some numerical examples to illustrate the results in Section 5 We ends our paper by conclusions 6 .

\section{HJB EQUATION}

For nonnegative $v \in \mathcal{C}^{2}$ with our optimization problem we associate the following Hamilton-JacobiBellman (HJB) equation:

$$
\max _{0 \leq q \leq 1, c \geq 0}\left\{(\theta-\eta q) a v^{\prime}(x)+\frac{1}{2} b^{2}(1-q)^{2} v^{\prime \prime}(x)-c v^{\prime}(x)+u(c)-\beta v(x)\right\}=0,
$$

with the boundary condition

$$
v(0)=0
$$

see Fleming and Soner [15] for a beautiful overview. From now on we look only for those solutions $v$ of above $\mathrm{HJB}$ equation that can be equal to the value function $V$. In other words, we exclude those solutions that for any reasons cannot be a value function.

We start from the classical Verification Lemma. 
Lemma 1. Suppose that $v(x) \in C^{2}$ is a nonnegative solution of the HJB equation (2.5) with the boundary condition (2.6). Then $v(x) \geq V(x)$ on $(0, \infty)$ where $V$ is the value function defined in (1.3).

Proof. Fix $x>0$ and choose a pair of admissible strategies $c(\cdot)$ and $q(\cdot)$. Select $0<\xi_{1}<x<\xi_{2}$.

Define

$$
\tau_{x}=\inf \left\{t \geq 0: X_{t}^{\pi}=x\right\}
$$

and

$$
w(t, x)=e^{-\beta t} v(x) .
$$

By applying Itô's formula to the process $w\left(t, X_{t}^{\pi}\right)$, we obtain

$$
\begin{aligned}
& w\left(t \wedge \tau_{\xi_{1}} \wedge \tau_{\xi_{2}}, X_{t \wedge \tau_{\xi_{1}} \wedge \tau_{\xi_{2}}}\right) \\
& =v(x)+\int_{0}^{t \wedge \tau_{\xi_{1}} \wedge \tau_{\xi_{2}}} e^{-\beta s}\left\{\left(\theta-\eta q_{s}\right) a v^{\prime}\left(X_{s}^{\pi}\right)+\frac{1}{2} b^{2}\left(1-q_{s}\right)^{2} v^{\prime \prime}\left(X_{s}^{\pi}\right)-c_{s} v^{\prime}\left(X_{s}^{\pi}\right)-\beta v\left(X_{s}^{\pi}\right)\right\} d s \\
& \quad+\int_{0}^{t \wedge \tau_{\xi_{1}} \wedge \tau_{\xi_{2}}} e^{-\beta s} v^{\prime}\left(X_{s}^{\pi}\right) b\left(1-q_{s}\right) d B_{s} \\
& \leq v(x)-\int_{0}^{t \wedge \tau_{\xi_{1}} \wedge \tau_{\xi_{2}}} e^{-\beta s} u\left(c_{s}\right) d s+\int_{0}^{t \wedge \tau_{\xi_{1}} \wedge \tau_{\xi_{2}}} e^{-\beta s} v^{\prime}\left(X_{s}^{\pi}\right) b\left(1-q_{s}\right) d B_{s},
\end{aligned}
$$

where the inequality is derived from the HJB equation (2.5). Since the reinsurance strategy $q_{s}$ lies between 0 and 1 , therefore the stochastic integration term of (2.7) is actually a martingale. Taking expectation on both sides of (2.7), we get

$$
\mathbb{E}_{x}\left[w\left(t \wedge \tau_{\xi_{1}} \wedge \tau_{\xi_{2}}, X_{t \wedge \tau_{\xi_{1}} \wedge \tau_{\xi_{2}}}^{\pi}\right)+\int_{0}^{t \wedge \tau_{\xi_{1}} \wedge \tau_{\xi_{2}}} e^{-\beta s} u\left(c_{s}\right) d s\right] \leq v(x) .
$$

Now let $\xi_{1} \downarrow 0, \xi_{2} \rightarrow \infty$ and $t \rightarrow \infty$, then $t \wedge \tau_{\xi_{1}} \wedge \tau_{\xi_{2}} \uparrow \tau$ almost surely. Thus by applying Fatou's lemma for the first term of the expectation in $(2.8)$, we have

$$
\liminf \mathbb{E}_{x} w\left(t \wedge \tau_{\xi_{1}} \wedge \tau_{\xi_{2}}, X_{t \wedge \tau_{\xi_{1}} \wedge \tau_{\xi_{2}}}^{\pi}\right) \geq \mathbb{E}_{x}\left[\liminf w\left(t \wedge \tau_{\xi_{1}} \wedge \tau_{\xi_{2}}, X_{t \wedge \tau_{\xi_{1}} \wedge \tau_{\xi_{2}}}^{\pi}\right)\right]=0,
$$

and by applying the monotone convergence theorem, the second term of the expectation in (2.8) converges to

$$
\mathbb{E}_{x}\left[\int_{0}^{\tau} e^{-\beta s} u\left(c_{s}\right) d s\right] .
$$

Hence, we can conclude that:

$$
\mathbb{E}_{x}\left[\int_{0}^{\tau} e^{-\beta s} u\left(c_{s}\right) d s\right] \leq v(x) .
$$

Maximization over all admissible strategies $c(\cdot)$ and $q(\cdot)$ gives the final result.

Note that if (1.4) holds, then supremum in (2.5) without constraints is realized for

$$
q(x)=1+\frac{\eta a}{b^{2}} \frac{v^{\prime}(x)}{v^{\prime \prime}(x)}
$$

and

$$
c(x)=\left(v^{\prime}(x)\right)^{-\frac{1}{1-p}} .
$$

Taking strategy $\pi=\left(q\left(X_{t}^{\pi}\right), c\left(X_{t}^{\pi}\right)\right)$ makes process $X_{t}^{\pi}$ to be a diffusion.

Denote:

$$
\alpha=1+\frac{2 b^{2} \beta}{\eta^{2} a^{2}} .
$$


We will solve our optimization problem under two family of assumptions:

Assumption 1. $\eta>\theta, \alpha(1-p)>1$ and $(2-\alpha(1-p)) \eta-2 \theta<0$

which is for non-cheap reinsurance and

Assumption 2. $\eta=\theta$ and $\alpha(1-p)>1$

which is for cheap reinsurance.

For the optimal value function $V$, we can also derive the following property, a similar result can be found in Choulli et al. [12].

Lemma 2. The optimal value function $V$ is increasing and strictly concave.

Proof. It is obvious to see that $V$ is increasing. To prove the concavity we will follow the idea of the proof of [12, Prop.2]. From the dynamic programming principle, we know $V(x)$ satisfies the following equality

$$
V(x)=\max _{\pi \in \Pi} \mathbb{E}_{x}\left[\int_{0}^{\tau_{y}} e^{-\beta s} u\left(c_{s}\right) d s+e^{-\beta \tau_{y}} V(y)\right],
$$

where $0<y<x$ and

$$
\tau_{y}=\inf \left\{t: X_{t}^{\pi}=y\right\} .
$$

For $h>0$, let $\Pi^{h}$ be the set of strategies $\pi$ such that

$$
\int_{0}^{\zeta}\left(\theta-\eta q_{s}\right) a d s+\int_{0}^{\zeta} b\left(1-q_{s}\right) d B_{s}-\int_{0}^{\zeta} c_{s} d s=-h
$$

on the set $\{\zeta<\infty\}$, where $\zeta$ is a stopping time defined by

$$
\zeta=\inf \left\{t \geq 0: \int_{0}^{t}\left(\theta-\eta q_{s}\right) a d s+\int_{0}^{t} b\left(1-q_{s}\right) d B_{s}-\int_{0}^{t} c_{s} d s=-h\right\} .
$$

By putting $h=x-y$ (hence $y=x-h$ ) from (2.12) we obtain that

$$
V(x)=\max _{\pi \in \Pi^{h}} \mathbb{E}_{x}\left[\int_{0}^{\zeta} e^{-\beta s} u\left(c_{s}\right) d s+e^{-\beta \zeta} V(y)\right] .
$$

Then

$$
V(x)-V(x-h)=\max _{\pi \in \Pi^{h}} \mathbb{E}_{x}\left[\int_{0}^{\zeta} e^{-\beta s} u\left(c_{s}\right) d s+\left(e^{-\beta \zeta}-1\right) V(x-h)\right] .
$$

Since $e^{-\beta \zeta}<1$ and $V(x)$ is nondecreasing function of $x$, the right-hand side of (2.14) is a decreasing function of $x$. Thus $V(x)-V(x-h)$ is decreasing in $x$. Hence, $V^{\prime}(x)$ is also decreasing and $V$ is strictly concave.

As the analysis above, the idea to solve the original optimization problem is to first find an increasing, concave and nonnegative solution $v$ of HJB equation (2.5) with the boundary condition (2.6), and then construct strategies such that the derived function $v$ can be realized by them. Thus the reverse inequality of Lemma 1 holds for this candidate solution $v$. Hence, $v$ is indeed the value function we are looking for. 


\section{NON-CHEAP REINSURANCE}

We will show that there exists a point $x^{*}$ such that

$$
q(x)=0 \quad \text { for } x>x^{*} .
$$

It implies that when the wealth of the insurance company is larger than $x^{*}$ then the optimal strategy will be to not reinsure the arrive claims. In other words, the insurance company can afford to cover all the claims by itself. Therefore, we analyze the value function in two intervals $\left(0, x^{*}\right]$ and $\left(x^{*}, \infty\right)$, respectively. Let

$$
\begin{aligned}
& B=-\frac{2 b^{2}}{\eta^{2} a^{2}} \frac{1-p}{1-\alpha+\alpha p}>0, \\
& D=\frac{2 b^{2}}{\alpha \eta^{2} a}(\eta-\theta)>0 .
\end{aligned}
$$

Proposition 3. Assume Assumption 1 Then on $\left(0, x^{*}\right]$ the function $v$ solving (2.5) is given by

$$
v(x)=\frac{e^{-\xi}}{\beta}\left[-(\eta-\theta) a+\frac{\eta^{2} a^{2}}{2 b^{2}} B e^{\frac{\xi}{1-p}}+\frac{\eta^{2} a^{2}}{2 b^{2}} D+\frac{1-p}{p} e^{\frac{\xi}{1-p}}\right],
$$

where $B$ and $D$ are given in (3.16) and (3.17) and $\xi=g^{-1}(x)$, where $g^{-1}$ is the inverse of function $g$ :

$$
g(\xi)=(1-p) B e^{\frac{\xi}{1-p}}+D \xi+Q_{1},
$$

for $Q_{1}=-(1-p) B e^{\frac{\xi_{0}}{1-p}}-D \xi_{0}$ and

$$
\xi_{0}=(1-p) \ln \frac{(\eta-\theta)(\alpha-1-\alpha p) a p}{\alpha(1-p)^{2}} .
$$

Above,

$$
x^{*}=g\left(\xi^{*}\right)
$$

where

$$
\xi^{*}=(1-p) \ln \left(\frac{b^{2}-\eta a D}{\eta a B}\right) .
$$

Proof. Substituting (2.9) and (2.10) into (2.5) gives:

$$
-(\eta-\theta) a v^{\prime}(x)-\frac{\eta^{2} a^{2}}{2 b^{2}} \frac{\left(v^{\prime}(x)\right)^{2}}{v^{\prime \prime}(x)}+\frac{1-p}{p}\left(v^{\prime}(x)\right)^{-\frac{p}{1-p}}-\beta v(x)=0 .
$$

We choose the following variable transform

$$
x=g(\xi), \quad v^{\prime}(g(\xi))=e^{-\xi} .
$$

Indeed, note that

$$
v^{\prime \prime}(g(\xi))=-\frac{e^{-\xi}}{g^{\prime}(\xi)}
$$

Plugging it into (3.23) produces

$$
-(\eta-\theta) a e^{-\xi}+\frac{\eta^{2} a^{2}}{2 b^{2}} e^{-\xi} g^{\prime}(\xi)+\frac{1-p}{p} e^{\frac{p}{1-p} \xi}-\beta v(g(\xi))=0 .
$$

Taking derivatives with $\xi$ and collecting terms, we derive

$$
\frac{\eta^{2} a^{2}}{2 b^{2}} g^{\prime \prime}(\xi)-\left(\frac{\eta^{2} a^{2}}{2 b^{2}}+\beta\right) g^{\prime}(\xi)+(\eta-\theta) a+e^{\frac{\xi}{1-p}}=0 .
$$


Denote $h(\xi)=g^{\prime}(\xi)$. Then the above equation can be rewritten as follows:

$$
h^{\prime}(\xi)-\alpha h(\xi)+\frac{2 b^{2}}{\eta^{2} a}(\eta-\theta)+\frac{2 b^{2}}{\eta^{2} a^{2}} e^{\frac{\xi}{1-p}}=0
$$

for some $Q=h(0)$. Solving above ordinary differential equation (ODE) we can observe that

$$
h(\xi)=Q e^{\alpha \xi}-\int_{0}^{\xi} \frac{2 b^{2}}{\eta^{2} a}(\eta-\theta) e^{\alpha(\xi-u)} d u-\int_{0}^{\xi} \frac{2 b^{2}}{\eta^{2} a^{2}} e^{\alpha \xi} e^{\left(\frac{1}{1-p}-\alpha\right) u} d u .
$$

Careful calculation and rearranging all terms give

$$
h(\xi)=A e^{\alpha \xi}+B e^{\frac{\xi}{1-p}}+D
$$

for

$$
A=Q-\frac{2 b^{2}}{\alpha \eta^{2} a}(\eta-\theta)+\frac{2 b^{2}}{\eta^{2} a^{2}} \frac{1-p}{1-\alpha+\alpha p} .
$$

In view of (3.25) and the concavity of $v$, the value of $A$ should be nonnegative. Then

$$
h(\xi)=A e^{\alpha \xi}+B e^{\frac{\xi}{1-p}}+D>0 .
$$

Recall that $h(\xi)=g^{\prime}(\xi)$ and (3.26), we get

$$
\begin{aligned}
v(x) & =\frac{e^{-\xi}}{\beta}\left[-(\eta-\theta) a+\frac{\eta^{2} a^{2}}{2 b^{2}} g^{\prime}(\xi)+\frac{1-p}{p} e^{\frac{\xi}{1-p}}\right] \\
& =\frac{e^{-\xi}}{\beta}\left[-(\eta-\theta) a+\frac{\eta^{2} a^{2}}{2 b^{2}} A e^{\alpha \xi}+\frac{\eta^{2} a^{2}}{2 b^{2}} B e^{\frac{\xi}{1-p}}+\frac{\eta^{2} a^{2}}{2 b^{2}} D+\frac{1-p}{p} e^{\frac{\xi}{1-p}}\right] .
\end{aligned}
$$

Note that if we allow a general values of $q_{t}$ without constrains then we only increase the value function giving us the possibility of considering the limit as $x \rightarrow \infty$. From (3.24), (3.30) and (3.31), if $A$ is strictly positive, we get

$$
v(x) \sim K_{0} x^{\frac{\alpha-1}{\alpha}},
$$

where $K_{0}$ is some fixed constant and $a(x) \sim b(x)$ means that $\lim _{x \rightarrow \infty} a(x) / b(x)=1$. However, the following upper bound

$$
V(x) \leq \mathbb{E}\left[\int_{0}^{\infty} e^{-\beta s} u\left(x+(\theta+1) a s+b B_{s}^{+}\right) d s\right]
$$

shows that there exists constant $K>0$ such that

$$
V(x) \leq K x^{p} .
$$

Therefore, if we want to have $v(x)=V(x)$, by Assumption 1 we must choose $A$ to be equal zero. Thus we obtain the representation of the function $v$ given in (3.18).

In the following, we will derive the value of $\xi_{0}$ which is given in (3.20) and satisfies $g\left(\xi_{0}\right)=0$. Indeed, if we substitute $\xi_{0}$ into 3.31 recalling that $v(0)=0$, then:

$$
-(\eta-\theta) a+\frac{\eta^{2} a^{2}}{2 b^{2}} g^{\prime}\left(\xi_{0}\right)+\frac{1-p}{p} e^{\frac{\xi_{0}}{1-p}}=0 .
$$

Since $g^{\prime}\left(\xi_{0}\right)=B e^{\frac{\xi_{0}}{1-p}}+D$ we obtain expression (3.20). Note that $h(\xi)=g^{\prime}(\xi)$ and $A=0$ we get (3.19) from (3.30).

We will prove that $0<q(x) \leq 1$ as it should be from the construction of the reinsurance policy. Substituting $v^{\prime}(x)=e^{-\xi}$ and $v^{\prime \prime}(x)=-e^{-\xi} / g^{\prime}(\xi)$ into (2.9), we get

$$
q(x)=1-\frac{\eta a}{b^{2}} g^{\prime}(\xi) \leq 1 .
$$


To satisfy required condition $q(x)>0$ we need to have the following inequality

$$
\frac{\eta a}{b^{2}} g^{\prime}(\xi)<1
$$

satisfied. We will show that $\xi^{*}$ given in (3.22) is the unique solution of the equation:

$$
\frac{\eta a}{b^{2}} g^{\prime}(\xi)=1 \text {. }
$$

It is easily verified that $\xi^{*}$ solves the above equation. Moreover, since

$$
g^{\prime \prime}(\xi)=\frac{B}{1-p} e^{\frac{\xi}{1-p}}>0,
$$

the function $g^{\prime}(\xi)$ is strictly increasing. Hence, $\xi^{*}$ solves above equation uniquely if

$$
\frac{\eta a}{b^{2}} g^{\prime}\left(\xi_{0}\right)<1
$$

Plugging (3.20) into the left side of inequality (3.33) we can conclude that

$$
\begin{aligned}
\frac{\eta a}{b^{2}} g^{\prime}\left(\xi_{0}\right) & =\frac{2}{\eta a}\left[-\frac{1-p}{1-\alpha+\alpha p} e^{\frac{\xi_{0}}{1-p}}+\frac{(\eta-\theta) a}{\alpha}\right] \\
& =\frac{2}{\eta a}\left[-\frac{1-p}{1-\alpha+\alpha p} \frac{(\eta-\theta)(\alpha-1-\alpha p) a p}{\alpha(1-p)^{2}}+\frac{(\eta-\theta) a}{\alpha}\right] \\
& =\frac{2(\eta-\theta)}{\alpha \eta(1-p)} .
\end{aligned}
$$

Therefore, if $2(\eta-\theta) /(\alpha \eta(1-p))<1$, that is $(2-\alpha(1-p)) \eta-2 \theta<0$, then (3.33) holds true.

Finally, we will check that $v$ is indeed a $C^{2}$ function solving the HJB equation (2.5) on $\left(0, x^{*}\right]$. From (3.31) we have

$$
\begin{aligned}
v^{\prime}(x) & =v^{\prime}(\xi) \cdot \frac{1}{g^{\prime}(\xi)} \\
& =\left\{-\frac{e^{-\xi}}{\beta}\left[-(\eta-\theta) a+\frac{\eta^{2} a^{2}}{2 b^{2}} g^{\prime}(\xi)+\frac{1-p}{p} e^{\frac{\xi}{1-p}}\right]+\frac{e^{-\xi}}{\beta}\left[\frac{\eta^{2} a^{2}}{2 b^{2}} g^{\prime \prime}(\xi)+\frac{1}{p} e^{\frac{\xi}{1-p}}\right]\right\} \cdot \frac{1}{g^{\prime}(\xi)} \\
& =\frac{e^{-\xi}}{\beta g^{\prime}(\xi)}\left\{(\eta-\theta) a-\frac{\eta^{2} a^{2}}{2 b^{2}} g^{\prime}(\xi)+e^{\frac{\xi}{1-p}}+\frac{\eta^{2} a^{2}}{2 b^{2}} g^{\prime \prime}(\xi)\right\} \\
& =e^{-\xi}
\end{aligned}
$$

where the last equality is obtained by 3.27 , and

$$
v^{\prime \prime}(x)=-\frac{e^{-\xi}}{g^{\prime}(\xi)}<0
$$

Therefore, $v$ is increasing and strictly concave. Substituting the values of $v^{\prime}$ and $v^{\prime \prime}$ into the left hand side of $\mathrm{HJB}$ equation (2.5), we have

$$
\begin{aligned}
& \max _{0 \leq q \leq 1, c \geq 0}\left\{(\theta-\eta q) a v^{\prime}(x)+\frac{1}{2} b^{2}(1-q)^{2} v^{\prime \prime}(x)-c v^{\prime}(x)+u(c)-\beta v(x)\right\} \\
& =\max _{0 \leq q \leq 1, c \geq 0}\left\{(\theta-\eta q) a e^{-\xi}-\frac{1}{2} b^{2}(1-q)^{2} \frac{e^{-\xi}}{g^{\prime}(\xi)}-c e^{-\xi}+u(c)-\beta v(x)\right\} .
\end{aligned}
$$

Since $u(\cdot)$ is an increasing and concave function and $0<g^{\prime}(\xi) \leq b^{2} / \eta a$ for $\xi_{0}<\xi \leq \xi^{*}$, therefore the maximizers $q^{*}$ and $c^{*}$ are given by

$$
\begin{aligned}
& q^{*}(x)=1-\frac{\eta a}{b^{2}} g^{\prime}(\xi), \\
& c^{*}(x)=e^{\frac{\xi}{1-p}}
\end{aligned}
$$


with $x=g(\xi)$. Hence, the equation (3.34) can be rewritten as follows:

$$
-(\eta-\theta) a e^{-\xi}+\frac{\eta^{2} a^{2}}{2 b^{2}} e^{-\xi} g^{\prime}(\xi)+\frac{1-p}{p} e^{\frac{p \xi}{1-p}}-\beta v(x)=0 .
$$

The verification of (3.35) is straightforward involving (3.18) and (3.19).

Remark 4. To solve the equation (3.23) we can also use the Legendre transform method. That is, we define

$$
\hat{v}(z)=\max _{x>0}\{v(x)-x z\}
$$

where the maximizing value of $x$ in (3.36) is the inverse function $I(z)$ of $v^{\prime}$. Then $v(x)$ can be recovered by

$$
v(x)=\min _{z>0}\{\hat{v}(z)+x z\}
$$

and the minimizing value of $z$ in (3.37) equals $v^{\prime}(x)$. Substituting $x=I(z)$ into (3.23) produces:

$$
\frac{\eta^{2} a^{2}}{2 b^{2}} z^{2} \hat{v}^{\prime \prime}(z)+\beta z \hat{v}^{\prime}(z)-\beta \hat{v}(z)+\frac{1-p}{p} z^{-\frac{p}{1-p}}-(\eta-\theta) a z=0
$$

Taking derivatives on both sides of (3.38) gives:

$$
\frac{\eta^{2} a^{2}}{2 b^{2}} z^{2} \hat{v}^{\prime \prime \prime}(z)+\left(\frac{\eta^{2} a^{2}}{b^{2}}+\beta\right) z \hat{v}^{\prime \prime}(z)-z^{-\frac{1}{1-p}}-(\eta-\theta) a=0 .
$$

Denote $h_{1}(z)=\hat{v}^{\prime}(z), z=e^{-t}$. Then (3.39) can be rewritten as

$$
\frac{\eta^{2} a^{2}}{2 b^{2}} h_{1}^{\prime \prime}(t)-\left(\frac{\eta^{2} a^{2}}{2 b^{2}}+\beta\right) h_{1}^{\prime}(t)-e^{\frac{t}{1-p}}-(\eta-\theta) a=0 .
$$

By substituting $h_{2}(t)=h_{1}^{\prime}(t)$ into (3.40) we get

$$
h_{2}^{\prime}(t)-\alpha h_{2}(t)-\frac{2 b^{2}(\eta-\theta)}{\eta^{2} a}-\frac{2 b^{2}}{\eta^{2} a^{2}} e^{\frac{t}{1-p}}=0 .
$$

The solution of (3.41) is then given by:

$$
h_{2}(t)=-A e^{\alpha t}-B e^{\frac{t}{1-p}}-D .
$$

Moreover, from (3.23) and the definition of the Legendre transform, we can find that

$$
\begin{aligned}
\beta v(x) & =-(\eta-\theta) a z+\frac{\eta^{2} a^{2}}{2 b^{2}} z^{2} \hat{v}^{\prime \prime}(z)+\frac{1-p}{p} z^{-\frac{p}{1-p}} \\
& =-(\eta-\theta) a e^{-t}-\frac{\eta^{2} a^{2}}{2 b^{2}} e^{-t} h_{2}(t)+\frac{1-p}{p} e^{\frac{p t}{1-p}},
\end{aligned}
$$

which coincides with 3.31).

We will now consider the value function $v$ on the interval $\left(x^{*}, \infty\right)$ on which $q^{*}(x) \equiv 0$. It is easy to verify that under the assumption that $q(x)=0$ on $\left(x^{*}, \infty\right)$ the HJB equation is equivalent to the following equation:

$$
\theta a v^{\prime}(x)+\frac{1}{2} b^{2} v^{\prime \prime}(x)+\frac{1-p}{p}\left(v^{\prime}(x)\right)^{-\frac{p}{1-p}}-\beta v(x)=0
$$

with the boundary conditions

$$
\begin{gathered}
v^{\prime}\left(x^{*}\right)=e^{-\xi^{*}}, \\
\frac{v^{\prime}\left(x^{*}\right)}{v^{\prime \prime}\left(x^{*}\right)}=-\frac{b^{2}}{\eta a} .
\end{gathered}
$$

It remains now to verify that if $v$ solves the equation (3.43), then the optimal reinsurance proportion indeed equals zero. 
Proposition 5. Assume that Assumption 1 holds. Suppose also that $v$ on $\left(x^{*}, \infty\right)$ solves (3.43)-(3.45). Then $v^{\prime}(x)>0$ and $v^{\prime \prime}(x)<0$ on $\left(x^{*}, \infty\right)$ and the maximum in

$$
\max _{0 \leq q \leq 1}\left\{(\theta-\eta q) a v^{\prime}(x)+\frac{1}{2} b^{2}(1-q)^{2} v^{\prime \prime}(x)\right\}=0
$$

is attained for $q=0$ in this case.

Proof. We first show that $v^{\prime}(x)>0$. Assume a contrario that there exists a point $\breve{x}$ on $\left(x^{*}, \infty\right)$ such that $v^{\prime}(\breve{x})<0$. This implies that we can find a point $\ddot{x}, x^{*}<\ddot{x}<\breve{x}$ satisfying $v^{\prime}(\ddot{x})=0$. Then

$$
v^{\prime \prime}(\ddot{x}-)=\lim _{x \uparrow \ddot{x}} \frac{v^{\prime}(x)-v^{\prime}(\ddot{x})}{x-\ddot{x}}<0 .
$$

Substituting $\ddot{x}$ into equation (3.43) gives:

$$
\frac{1}{2} b^{2} v^{\prime \prime}(\ddot{x}-)=\beta v(\ddot{x})>0
$$

which is a contradiction. Hence $v$ is indeed an increasing function.

Function $v$ is also concave, that is, $v^{\prime \prime}(x)<0$. Indeed, denote

$$
y(v)=v^{\prime}(x)>0 .
$$

Then $v^{\prime \prime}(x)=y(v) y^{\prime}(v)$ and plugging it into (3.43) produces

$$
\theta a y(v)+\frac{1}{2} b^{2} y(v) y^{\prime}(v)+\frac{1-p}{p} y(v)^{-\frac{p}{1-p}}-\beta v=0
$$

with the boundary conditions $y^{\prime}\left(v^{*}\right)=-\eta a / b^{2}<0$ and $y\left(v^{*}\right)=e^{-\xi^{*}}$. Moreover, the equation (3.47) can be rewritten as follows:

$$
y^{\prime}(v)=\frac{2}{b^{2}}\left(\beta \frac{v}{y(v)}-\theta a-\frac{1-p}{p} y(v)^{-\frac{1}{1-p}}\right) .
$$

According to Theorem 3 of Hubalek and Schachermayer [20], the differential equation (3.48) has precisely one decreasing convex solution. Therefore,

$$
y^{\prime}(v)<0 \quad \text { and } \quad y^{\prime \prime}(v)>0 .
$$

Hence, $v^{\prime \prime}(x)<0$.

Now we will show that (3.46) attaines maximum at zero, that is

$$
-\frac{\eta a}{b^{2}} \frac{v^{\prime}(x)}{v^{\prime \prime}(x)}>1
$$

or that

$$
y^{\prime}(v)>-\frac{\eta a}{b^{2}} .
$$

Denote

$$
q(v)=\left(\theta-\frac{\eta}{2}\right) a y(v)+\frac{1-p}{p} y(v)^{-\frac{p}{1-p}} .
$$

Since $y(v)$ satisfies equation (3.47), thus $q(v)$ can be rewritten as:

$$
q(v)=\beta v-\frac{\eta a}{2} y(v)-\frac{1}{2} b^{2} y(v) y^{\prime}(v) .
$$

Therefore demonstrating (3.51) is equivalent to showing that

$$
q(v)<\beta v \quad \text { for } v>v^{*} .
$$


Due to the boundary condition $y^{\prime}\left(v^{*}\right)=-\eta a / b^{2}$, we can observe that $q\left(v^{*}\right)=\beta v^{*}$. We also claim that

$$
q^{\prime}\left(v^{*}\right)<\beta
$$

Indeed,

$$
\begin{aligned}
q^{\prime}\left(v^{*}\right) & =\left(\theta-\frac{\eta}{2}\right) a y^{\prime}\left(v^{*}\right)-y\left(v^{*}\right)^{-\frac{1}{1-p}} y^{\prime}\left(v^{*}\right) \\
& =-\frac{\eta a^{2}}{b^{2}}\left(\theta-\frac{\eta}{2}\right)+\frac{\eta a}{b^{2}} e^{\frac{\xi^{*}}{1-p}} \\
& =-\frac{\eta a^{2}}{b^{2}}\left(\theta-\frac{\eta}{2}\right)+\frac{\eta a}{b^{2}} \frac{1}{B}\left(\frac{b^{2}}{\eta a}-D\right) \\
& =\beta-\frac{\eta a^{2}}{2 \alpha b^{2}(1-p)}(\eta \alpha-2(\eta-\theta)),
\end{aligned}
$$

where the second equality is obtained by substituting the values of $y\left(v^{*}\right)$ and $y^{\prime}\left(v^{*}\right)$. The third equality follows from the form of $\xi^{*}$ and the last equality is obtained by plugging the values of $B, D$ and $\alpha$. Now the inequality $\eta \alpha-2(\eta-\theta)>\alpha p \eta>0$ implies (3.53).

To prove (3.52), suppose a contrario that there exists $\tilde{v} \in\left(v^{*}, \infty\right)$ such that $q(\tilde{v})=\beta \tilde{v}$, that is $y^{\prime}(\tilde{v})=-\eta a / b^{2}$, and that $q(v)<\beta v$ on $\left(v^{*}, \tilde{v}\right)$. Since $q(\tilde{v})=\beta \tilde{v}$ and $q(v)<\beta v$ on $\left(v^{*}, \tilde{v}\right)$, we have $q^{\prime}(\tilde{v})>\beta$. This implies that

$$
\left(\theta-\frac{\eta}{2}\right) a y^{\prime}(\tilde{v})-y(\tilde{v})^{-\frac{1}{1-p}} y^{\prime}(\tilde{v})>\beta
$$

On the other hand, by taking derivatives with $v$ on both sides of (3.47) and by substituting the value of $\tilde{v}$, we obtain

$$
\theta a y^{\prime}(\tilde{v})+\frac{1}{2} b^{2}\left(y^{\prime}(\tilde{v})\right)^{2}+\frac{1}{2} b^{2} y(\tilde{v}) y^{\prime \prime}(\tilde{v})-y(\tilde{v})^{-\frac{1}{1-p}} y^{\prime}(\tilde{v})-\beta=0
$$

and therefore

$$
\theta a y^{\prime}(\tilde{v})-y(\tilde{v})^{-\frac{1}{1-p}} y^{\prime}(\tilde{v})=\beta-\frac{1}{2} b^{2}\left(y^{\prime}(\tilde{v})\right)^{2}-\frac{1}{2} b^{2} y(\tilde{v}) y^{\prime \prime}(\tilde{v}) .
$$

Now, substituting above identity into (3.54) gives

$$
y^{\prime \prime}(\tilde{v})<0
$$

because $y^{\prime}(\tilde{v})=-\eta a / b^{2}$. This is a contradiction with the fact that by (3.49) $y(v)$ is a decreasing convex function. This completes the proof.

The equation (3.43) satisfied by $v$ for $x>x^{*}$ is similar to equation (15) in Hubalek and Schachermayer [20]. Therefore, we can identify the asymptotic solution of the value function and the corresponding optimal strategies as it was also done in Hubalek and Schachermayer [20].

Theorem 6 . The asymptotic behaviors of $v(x), c(x), q(x)$, as $x \rightarrow \infty$, are given by:

$$
\begin{aligned}
& v(x) \sim\left(\frac{1-p}{\beta}\right)^{1-p} \frac{x^{p}}{p}, \\
& c(x) \sim \frac{\beta}{1-p} x, \\
& q(x) \equiv 0 .
\end{aligned}
$$

Summarizing Propositions 3,5 and Theorem 6 gives the first main result of this paper. 
Theorem 7. Assume Assumption 1 Then the value function $V(x)$ is given by

$$
V(x)= \begin{cases}\frac{e^{-\xi}}{\beta}\left[-(\eta-\theta) a+\frac{\eta^{2} a^{2}}{2 b^{2}} B e^{\frac{\xi}{1-p}}+\frac{\eta^{2} a^{2}}{2 b^{2}} D+\frac{1-p}{p} e^{\frac{\xi}{1-p}}\right], & 0<x \leq x^{*}, \\ v(x), & x>x^{*},\end{cases}
$$

with $B$ and D given in (3.16) and (3.17) and $x=g(\xi)$, where $g$ is defined in (3.19). Moreover, $x^{*}$ is given in (3.21) and $v(x)$ solves (3.43)- 3.45). The corresponding optimal dividend strategy is

$$
c(x)=\left(V^{\prime}(x)\right)^{-\frac{1}{1-p}}
$$

and the optimal reinsurance proportion is

$$
q(x)= \begin{cases}1-\frac{\eta a}{b^{2}} g^{\prime}(\xi), & 0<x \leq x^{*} \\ 0, & x>x^{*}\end{cases}
$$

Proof. Note that $c(x)$ and $q(x)$ have bounded continuous derivatives on $\left(0, x^{*}\right]$. For $x>x^{*}$, we have $q(x) \equiv 0$ and $c(x)$ is asymptotically equivalent to $\beta x /(1-p)$ as $x$ tends to $\infty$. Therefore, their derivatives are bounded on $\left(x^{*}, \infty\right)$. Hence, under the optimal strategy, the classical result in SDE guarantees the existence and uniqueness of the solution for SDE (1.2). Moreover, from Propositions 3 and 5 it follows that the right hand side $v(x)$ of (3.55) is a nonnegative and concave solution of HJB equation (2.5) with the boundary condition (2.6). Similarly to the proof of Lemma 1, applying Itô's formula to the process $w\left(t, X_{t}^{\pi^{*}}\right)$ with the corresponding strategies identified in Propositions 3 and 5

$$
\pi^{*}=\left(q_{t}^{*}, c_{t}^{*}\right)=\left(q\left(X_{t}^{\pi^{*}}\right), c\left(X_{t}^{\pi^{*}}\right)\right)
$$

produces:

$$
\begin{aligned}
& w\left(t \wedge \tau_{\xi_{1}} \wedge \tau_{\xi_{2}}, X_{t \wedge \tau_{\xi_{1}} \wedge \tau_{\xi_{2}}}^{\pi^{*}}\right) \\
& =v(x)+\int_{0}^{t \wedge \tau_{\xi_{1}} \wedge \tau_{\xi_{2}}} e^{-\beta s}\left\{\left(\theta-\eta q_{s}^{*}\right) a v^{\prime}\left(X_{s}^{\pi^{*}}\right)+\frac{1}{2} b^{2}\left(1-q_{s}^{*}\right)^{2} v^{\prime \prime}\left(X_{s}^{\pi^{*}}\right)-c_{s}^{*} v^{\prime}\left(X_{s}^{\pi^{*}}\right)-\beta v\left(X_{s}^{\pi^{*}}\right)\right\} d s \\
& \quad+\int_{0}^{t \wedge \tau_{\xi_{1}} \wedge \tau_{\xi_{2}}} e^{-\beta s} v^{\prime}\left(X_{s}^{\pi^{*}}\right) b\left(1-q_{s}^{*}\right) d B_{s}
\end{aligned}
$$

$$
=v(x)-\int_{0}^{t \wedge \tau_{\xi_{1}} \wedge \tau_{\xi_{2}}} e^{-\beta s} u\left(c_{s}^{*}\right) d s+\int_{0}^{t \wedge \tau_{\xi_{1}} \wedge \tau_{\xi_{2}}} e^{-\beta s} v^{\prime}\left(X_{s}^{\pi^{*}}\right) b\left(1-q_{s}^{*}\right) d B_{s},
$$

where the last equality is obtained by the HJB equation (2.5). Taking expectation on both sides of (3.56), we get

$$
\mathbb{E}_{x}\left[w\left(t \wedge \tau_{\xi_{1}} \wedge \tau_{\xi_{2}}, X_{t \wedge \tau_{\xi_{1}} \wedge \tau_{\xi_{2}}}^{\pi^{*}}\right)+\int_{0}^{t \wedge \tau_{\xi_{1}} \wedge \tau_{\xi_{2}}} e^{-\beta s} u\left(c_{s}^{*}\right) d s\right]=v(x) .
$$

We claim that $\left\{w\left(t, X_{t}^{\pi^{*}}\right)\right\}_{t \geq 0}$ is uniformly integrable. Then letting $\xi_{1} \downarrow 0, \xi_{2} \rightarrow \infty$ and $t \rightarrow \infty$, and by applying the dominated convergence theorem and the monotone convergence theorem, we have

$$
\mathbb{E}_{x}\left[\int_{0}^{\tau} e^{-\beta s} u\left(c_{s}^{*}\right) d s\right]=v(x) .
$$

Thus $v(x) \leq V(x)$ from the definition of the value function. Combining above inequality with the result obtained in Verification Lemma 1 completes the proof. In order to verify the uniformly integrability of 
$w\left(t, X_{t}^{*}\right)$, it is sufficient to show that the maximum function $w^{*}\left(T, X_{T}^{\pi^{*}}\right)=\sup _{0 \leq t \leq T} w\left(t, X_{t}^{\pi^{*}}\right)$ is integrable for each $T \geq 0$. By the concavity of $v$, we have

$$
v(x) \leq v^{\prime}(0) x
$$

Thus,

$$
\mathbb{E}_{x} w^{*}\left(T, X_{T}^{\pi^{*}}\right) \leq \mathbb{E}_{x}\left[\sup _{0 \leq t \leq T} v\left(X_{t}^{\pi^{*}}\right)\right] \leq v^{\prime}(0) \mathbb{E}_{x}\left[\sup _{0 \leq t \leq T} X_{t}^{\pi^{*}}\right] .
$$

We introduce now a new stochastic process $Y_{t}$ having the following dynamics:

$$
d Y_{t}=\theta a d t+b\left(1-q_{t}^{*}\right) d B_{t}
$$

with the same initial value as that of the reserve process $X_{t}^{\pi^{*}}$, that is, $Y_{0}=x$. Then one can observe that $\left\{Y_{t}\right\}_{0 \leq t \leq T}$ is a submartingale and $X_{t}^{\pi^{*}} \leq Y_{t}$ for any $t \geq 0$. We denote $Y_{T}^{*}=\sup _{0 \leq t \leq T} Y_{t}$. Then,

$$
\mathbb{E}_{x}\left[\sup _{0 \leq t \leq T} X_{t}^{\pi^{*}}\right] \leq \mathbb{E}_{x}\left(Y_{T}^{*}\right) \leq \sqrt{\mathbb{E}_{x}\left(Y_{T}^{*}\right)^{2}} \leq 2 \sqrt{\mathbb{E}_{x}\left(Y_{T}\right)^{2}} \leq K_{1} T+K_{2} \sqrt{T}<\infty,
$$

where $K_{1}, K_{2}$ are some fixed constants, the second inequality is obtained by Cauchy-Swartz inequality and the third inequality is derived by Doob's maximal inequality for submartingale. Therefore, in view of (3.57) and (3.59), we can conclude that $\left\{w\left(t, X_{t}^{\pi^{*}}\right)\right\}_{t \geq 0}$ is uniformly integrable. This completes our proof.

\section{CHEAP REINSURANCE}

In this section we consider the case $\eta=\theta$, that is that cheap reinsurance holds true. The corresponding HJB equation could be rewritten as follows:

$$
\max _{0 \leq q \leq 1, c \geq 0}\left\{\theta(1-q) a v^{\prime}(x)+\frac{1}{2} b^{2}(1-q)^{2} v^{\prime \prime}(x)-c v^{\prime}(x)+u(c)-\beta v(x)\right\}=0 .
$$

As argued in the previous section, we suppose that HJB equation (4.60) has an increasing concave solution $v$. Then, for $q$ without restriction, the left hand side of (4.60) attains its maximum at

$$
q(x)=1+\frac{\theta a}{b^{2}} \frac{v^{\prime}(x)}{v^{\prime \prime}(x)}
$$

and

$$
c(x)=\left(v^{\prime}(x)\right)^{-\frac{1}{1-p}} .
$$

Similarly, as in the non-cheap reinsurance case, we will find the point $x^{*}$ such that $q(x)=0$, for all $x>x^{*}$. Therefore, we have to consider here also two intervals $\left(0, x^{*}\right]$ and $\left(x^{*}, \infty\right)$. At the beginning we will analyze the case of $\left(0, x^{*}\right]$ on which $q(x)>0$. We recall the definition of $\alpha$ in (2.11) with $\eta=\theta$. Let

$$
x^{*}=\frac{b^{2}(1-p)}{\theta a} .
$$

Proposition 8. Assume that Assumption 2holds. Then for $x \in\left(0, x^{*}\right]$,

$$
v(x)=M \frac{x^{p}}{p},
$$

where

$$
M=\left[\frac{\beta}{1-p}-\frac{\theta^{2} a^{2}}{2 b^{2}} \frac{p}{(1-p)^{2}}\right]^{-(1-p)}
$$


Proof. Note that (4.60) can be rewritten as follows:

$$
-\frac{\theta^{2} a^{2}}{2 b^{2}} \frac{\left(v^{\prime}(x)\right)^{2}}{v^{\prime \prime}(x)}+\frac{1-p}{p}\left(v^{\prime}(x)\right)^{-\frac{p}{1-p}}-\beta v(x)=0 .
$$

The general solution of ODE [4.64) is given by (see Merton [28] for details):

$$
v(x)=\frac{M(x-N)^{p}}{p}+Q .
$$

Then

$$
v^{\prime}(x)=M(x-N)^{p-1}, \quad v^{\prime \prime}(x)=-M(1-p)(x-N)^{p-2} .
$$

Plugging it back into (4.64) produces $Q=0$ and $M$ given in (4.63). Note that $\alpha>1 /(1-p)$, that is, $1+2 b^{2} \beta / \theta^{2} a^{2}>1 /(1-p)$, which implies that $\beta>\theta^{2} a^{2} p /\left(2 b^{2}(1-p)\right)$. Hence, $M>0$. Finally, from the boundary condition $v(0)=0$ we get $N=0$. Thus the proof is completed.

We will now consider the interval $\left(x^{*}, \infty\right)$ on which we will have $q(x) \equiv 0$. Similarly as the non-cheap reinsurance case, from HJB equation (4.60) we can derive the equation satisfied by the value function on this interval:

$$
\theta a v^{\prime}(x)+\frac{1}{2} b^{2} v^{\prime \prime}(x)+\frac{1-p}{p}\left(v^{\prime}(x)\right)^{-\frac{p}{1-p}}-\beta v(x)=0
$$

with the boundary conditions

$$
\begin{aligned}
v^{\prime}\left(x^{*}\right) & =M\left(x^{*}\right)^{p-1}, \\
\frac{v^{\prime}\left(x^{*}\right)}{v^{\prime \prime}\left(x^{*}\right)} & =-\frac{x^{*}}{1-p} .
\end{aligned}
$$

Note that the second-order ODE (4.65) is the same as ODE (3.43) except the two boundary conditions. In the same way as in the proof of Proposition 5 one can verify that $q(x)=0$ for $x>x^{*}$. To do it one has to prove inequality

$$
-\frac{\theta a}{b^{2}} \frac{v^{\prime}(x)}{v^{\prime \prime}(x)}>1
$$

with $v(x)=M x^{p} / p$ as a counterpart of (3.50).

Proposition 9. Assume Assumptions 2 Suppose $v$ on $\left(x^{*}, \infty\right)$ solves 4.65)-4.67). Then $v$ satisfies $v^{\prime}(x)>0$ and $v^{\prime \prime}(x)<0$ and maximum in

$$
\max _{0 \leq q \leq 1}\left\{\theta(1-q) a v^{\prime}(x)+\frac{1}{2} b^{2}(1-q)^{2} v^{\prime \prime}(x)\right\}=0
$$

is attained for $q=0$ in this case.

Remark 10. Since (4.65) is the same as (3.43), we will have the same asymptotic results for the cheap reinsurance case as that for the non-cheap reinsurance case.

Based on the above analysis, we give the second main result of this paper.

Theorem 11. Assume Assumption 2 The value function $V(x)$ is given by

$$
V(x)= \begin{cases}M \frac{x^{p}}{p}, & 0<x \leq x^{*}, \\ v(x), & x>x^{*},\end{cases}
$$


with $x^{*}$ and $M$ in (4.62) and (4.63), respectively, and $v(x)$ solves 4.65)-4.67). The corresponding optimal dividend strategy is

$$
c(x)=\left(V^{\prime}(x)\right)^{-\frac{1}{1-p}}
$$

and the optimal reinsurance proportion is

$$
q(x)= \begin{cases}1-\frac{\theta a}{b^{2}(1-p)} x, & 0<x \leq x^{*}, \\ 0, & x>x^{*} .\end{cases}
$$

Proof. Similarly like in the proof of Theorem 7 one can prove the existence and uniqueness of the solution for SDE (1.2) for the chosen optimal strategies. Furthermore, in order to confirm that the candidate solution $v(x)$ is indeed the value function, it is sufficient to verify that $\left\{w\left(t, X_{t}^{\pi^{*}}\right)\right\}_{0 \leq t \leq T}$ is uniformly integrable, where $\pi^{*}$ is the corresponding strategy given in Propositions 8 and 9 However, since $\lim _{x \rightarrow 0} v^{\prime}(x)=\infty$, we cannot use the proof of Theorem 7 directly and some further modification is required here. By (3.32) we can find a large constant $K$ such that for $x>0$,

$$
v(x) \leq K x^{p} .
$$

Then,

$$
\mathbb{E}_{x} w^{*}\left(T, X_{T}^{\pi^{*}}\right) \leq \mathbb{E}_{x}\left[\sup _{0 \leq t \leq T} v\left(X_{t}^{\pi^{*}}\right)\right] \leq K \mathbb{E}_{x}\left[\sup _{0 \leq t \leq T}\left(X_{t}^{\pi^{*}}\right)^{p}\right] \leq K \mathbb{E}_{x}\left[\left(Y_{T}^{*}\right)^{p}\right],
$$

where $Y_{t}$ is given in (3.58) and $Y_{t}^{*}$ is its maximal process. Moreover, since $p \in(0,1)$, we can find two positive integral such that $p \leq m / n$. Thus,

$$
\mathbb{E}_{x}\left[\left(Y_{T}^{*}\right)^{p}\right] \leq \mathbb{E}_{x}\left[\left(Y_{T}^{*}\right)^{m / n}\right] \leq\left\{\mathbb{E}_{x}\left(Y_{T}^{*}\right)^{2 m}\right\}^{\frac{1}{2 n}} \leq\left(\frac{2 m}{2 m-1}\right)^{\frac{m}{n}}\left\{\mathbb{E}_{x}\left(Y_{T}\right)^{2 m}\right\}^{\frac{1}{2 n}} \leq H_{1} T^{\frac{m}{n}}+H_{2} T^{\frac{m}{2 n}}<\infty,
$$

where $H_{1}, H_{2}$ are some fixed constants. Therefore, $\left\{w\left(t, X_{t}^{\pi^{*}}\right)\right\}_{0 \leq t \leq T}$ is uniformly integrable. This completes the proof.

\section{NUMERICAL EXAMPLES}

In this section, we provide numerical examples to demonstrate the results obtained in Sections 3 and 4 We focus on the effects of the power $p$ and the claim volatility $b$ on the optimal strategies and the threshold $x^{*}$ for both cheap and noncheap reinsurance cases.

In Figure 1 we plot the optimal dividend and optimal reinsurance proportion for surplus $x$ ranging from 0 to 50. In Figure 1(a), we consider the noncheap reinsurance case. According to Theorem 7 we find the optimal threshold $x^{*}$ equals to 11.2578 , which implies that the insurer will take all the claims without buying any reinsurance when the surplus is larger than 11.2578. From this graph we can also observe that the optimal dividend rate $c^{*}(x)$ is nearly linear increasing with the surplus $x$, while the optimal reinsurance proportion is decreasing with $x$. When its surplus is sufficiently small, the insurer will divert most of its risk incurred by claims to the reinsurer, which seems intuitively reasonable. By comparison, the cheap reinsurance case has been illustrated in Figure 1(b), which exhibits similar phenomenon as in Figure 1(a) In accordance with Theorem 11, in this case, the optimal threshold $x^{*}$ equals to 20.6250, which is larger than that in Figure 1 This happens because the premium paid to the reinsurer in the cheap reinsurance case is less than that paid in the noncheap one. This means that insurer will be more willing to divert the claim risk to the reinsurer in cheap reinsurance case even though its reserves are relatively large. 
Figure 2 displays the optimal dividend strategy and the optimal reinsurance proportion as the functions of $p \in[0,1]$ for fixed $x=5$. From this graph, we can observe that the optimal dividend rate increases with increasing $p$, while the optimal reinsurance proportion decreases. This phenomenon can be explained by noting that the parameter $1-p$ represents the risk aversion of the insurer, which indicates the insurer's attitude towards the risk. We also find that for a fixed $p$, the optimal dividend rate and the optimal reinsurance proportion in cheap reinsurance case are larger than those in noncheap one. The reason behind this property is that insurer usually pays less under the cheap reinsurance contract and it tends to transfer most of the risks to the reinsurer paying extra money for dividends.

Figure 3 shows the values of $x^{*}$ with respect of changing parameters $p$ and $b$. In Figure 3(a) we set $b=0.5$ and change the values of $p$. Note that $x^{*}$ decreases as $p$ increases. Because $p$ reflects the insurer's tolerance of the risks, as $p$ becomes larger, the insurer becomes less risk averse. In Figure 3(b) we fix $p=0.01$ and observe that $x^{*}$ increases as $b$ increases. Indeed, this is explained by the fact that the larger values of $b$ indicate that more risks will be taken by the insurer. In both Figures $3(\mathrm{a})$ and $3(\mathrm{~b})$ the values of $x^{*}$ in cheap reinsurance case are bigger than those in noncheap reinsurance case which coincides with the analysis demonstrated in Figure1.

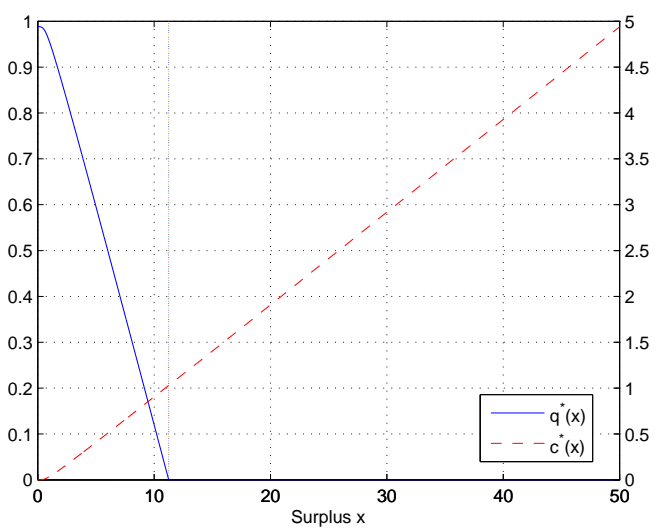

(a) $\eta=0.8, \theta=0.4$

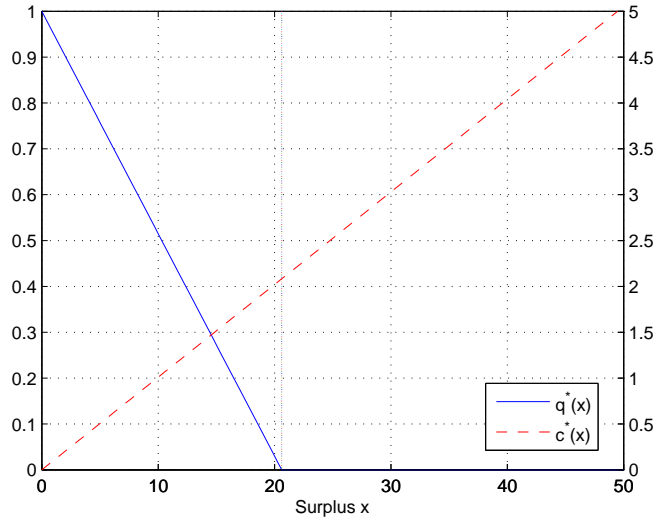

(b) $\eta=\theta=0.4$

FIGURE 1. $q^{*}(x)$ and $c^{*}(x)$ change with $x$ for $a=0.03, b=0.5, p=0.01, \beta=0.1$.

\section{CONCLUSIONS}

In this paper we manage to find the value function maximizing the discounted cumulative dividends payments paid up to ruin time where the strategy is based on choice of dividend payments and the proportion of the reinsurance policy. We analyzed only the Constant Relative Risk Aversion utility function. The future research will concern other utility functions. One can also choose more general stopping time of the regulated risk process. For example one can consider Parisian ruin time as it was done in Czarna and Palmowski [13]. Finally, it is also very interesting to incorporate so-called Gerber-Shiu function in the value function as it was realized in Avram et al. [5]. One can analyze the investments into risky assets as well, see Paulsen and Gjessing [29]. All of these problems are more complex and left for future research. 


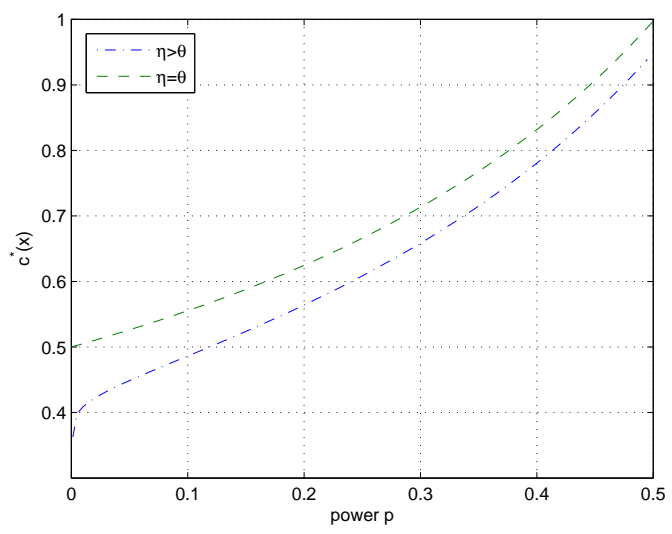

(a) $c^{*}(x)$ vs $p$

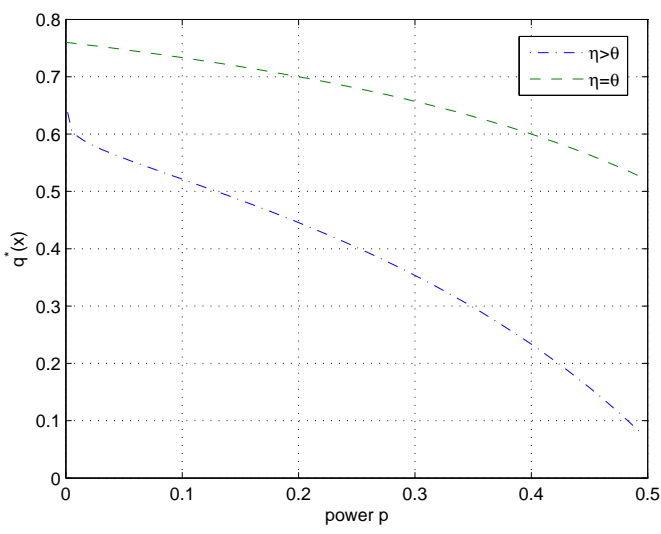

(b) $q^{*}(x)$ vs $p$

FIGURE 2. $c^{*}(x)$ and $q^{*}(x)$ change with $p$ for $a=0.03, b=0.5, \beta=0.1, \eta=0.8, \theta=$ $0.4, x=5$.

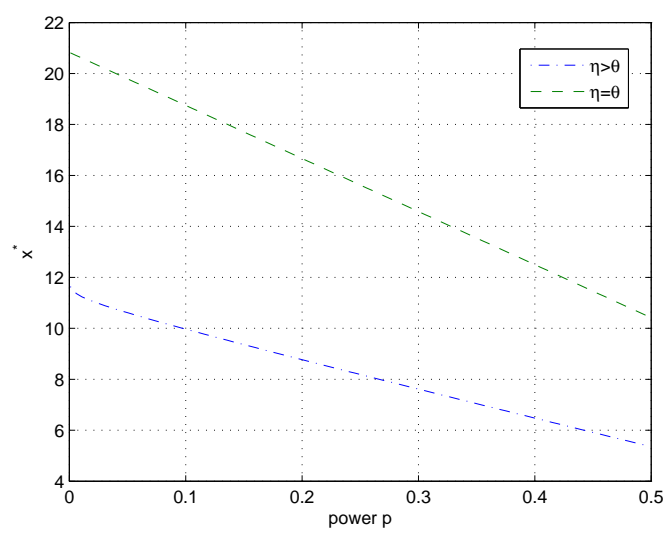

(a) $x^{*}$ vs $p$ for $b=0.5$

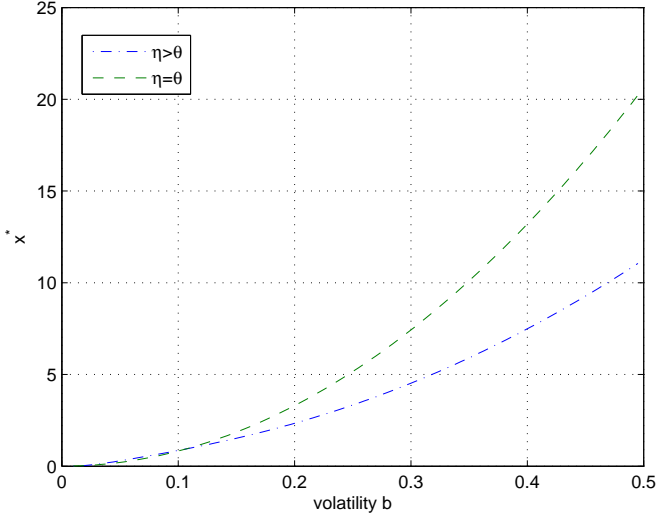

(b) $x^{*}$ vs $b$ for $p=0.01$

FIGURE 3. $x^{*}$ changes with $p$ and $b$ respectively for $a=0.03, \beta=0.1, \eta=0.8, \theta=0.4$.

\section{REFERENCES}

[1] Albrecher H. and Thonhauser S. (2009). Optimality Results for Dividend Problems in Insurance. RACSAM Rev. R. Acad. Cien. Serie A. Mat., 103(2):295-320.

[2] Asmussen, S., Højgaard, B., and Taksar, M. (2000). Optimal risk control and dividend distribution policies. Example of excess-of loss reinsurance for an insurance corporation. Finance and Stochastics, 4(3):299-324.

[3] Asmussen, S. and Taksar, M. (1997). Controlled diffusion models for optimal dividend pay-out. Insurance: Mathematics and Economics, 20(1):1-15.

[4] Avram, F., Palmowski, Z. and Pistorius, M.R. (2007). On the optimal dividend problem for a spectrally negative Lévy process. Ann. Appl. Probab., 17:156-180. 
[5] Avram, F., Palmowski, Z. and Pistorius, M.R. (2015). On Gerber-Shiu functions and optimal dividend distribution for a Lévy risk-process in the presence of a penalty function. Ann. Appl. Probab., 25(4):18681935.

[6] Azcue, P. and Muler, N. (2005). Optimal reinsurance and dividend distribution policies in the CramérLundberg model. Mathematical Finance, 15(2):261-308.

[7] Azcue, P. and Muler, N. (2015). Optimal dividend payment and regime switching in a compound poisson risk model. SIAM Journal on Control and Optimization, 53(5):3270-3298.

[8] Bai, L. and Guo, J. (2010). Optimal dividend payments in the classical risk model when payments are subject to both transaction costs and taxes. Scandinavian Actuarial Journal, 2010(1):36-55.

[9] Buhlmann, H. (1970). Mathematical Methods in Risk Theory. Springer-Verlag: Berlin.

[10] Chen, M., Peng, X. and Guo, J. (2013). Optimal dividend problem with a nonlinear regular-singular stochastic control. Insurance: Mathematics and Economics, 52(3):448-456.

[11] Cadenillas, A., Choulli, T., Taksar, M. and Zhang, L. (2006). Classical and impulse stochastic control for the optimization of the dividend and risk policies of an insurance firm. Mathematical Finance, 16:181202.

[12] Choulli, T., Taksar, M. and Zhou, X. Y. (2003). A diffusion model for optimal dividend distribution for a company with constraints on risk control. SIAM Journal on Control and Optimization, 41(6):1946-1979.

[13] Czarna, I. and Palmowski, Z. (2014). Dividend problem with Parisian delay for a spectrally negative Lévy risk process. Journal of Optimization Theory and Applications, 161:239-256.

[14] David Promislow, S. and Young, V. R. (2005). Minimizing the probability of ruin when claims follow Brownian motion with drift. North American Actuarial Journal, 9(3):110-128.

[15] Fleming, W. H. and Soner, H. M. (2006). Controlled Markov Processes and Viscosity Solutions, volume 25. Springer New York.

[16] Gerber, H.U. and Shiu, E.S.W. (2004). Optimal dividends: analysis with Brownian motion. North American Actuarial Journal, 8:1-20.

[17] Hipp, C. and Plum, M. (2000). Optimal investment for insurers. Insurance: Mathematics and Economics, 27:215-228.

[18] Højgaard and Taksar, M. (1999). Controlling risk exposure and dividends payout schemes: Insurance company example. Mathematical Finance, 9(2):153-182.

[19] Højgaard, B. and Taksar, M. (2004). Optimal dynamic portfolio selection for a corporation with controllable risk and dividend distribution policy. Quantitative Finance, 4(3):315-327.

[20] Hubalek, F. and Schachermayer, W. (2004). Optimizing expected utility of dividend payments for a Brownian risk process and a peculiar nonlinear ODE. Insurance: Mathematics and Economics, 34(2):193225.

[21] Jeanblanc, M. and Shiryaev, A.N. (1995). Optimization of the flow of dividends. Russian Math. Surveys, 50:257-277.

[22] Karatzas, I., Lehoczky, J.P., Sethi, S.P. and Shreve, S.E. (1986). Explicit Solution of a General Consumption/Investment Problem. Mathematics of Operations Research, 11(2):261-294.

[23] Kyprianou, A. and Palmowski, Z. (2007). Distributional study of De Finetti's dividend problem for a general Lévy insurance risk process. J. Appl. Probab., 44:428-443.

[24] Liang, Z. and Young, V. R. (2012). Dividends and reinsurance under a penalty for ruin. Insurance: Mathematics and Economics, 50(3):437-445.

[25] Loeffen, R. (2008). On optimality of the barrier strategy in de Finetti's dividend problem for spectrally negative Lévy processes. Ann. Appl. Probab., 18:1669-1680. 
[26] Loeffen, R. and Renaud, J-F. (2009). De Finetti's optimal dividends problem with an affine penalty function at ruin. Insurance: Mathematics and Economics, 46:98-108.

[27] Loeffen, R. (2009). An optimal dividends problem with transaction costs for spectrally negative Lévy processes. Insurance: Mathematics and Economics, 45(1):41-48.

[28] Merton, R.C. (1969) Lifetime portfolio selection under uncertainty: The continuous-time case. The review of Economics and Statistics, 51(3): 247-257.

[29] Paulsen, J. and Gjessing, H.K. (1997). Optimal choice of dividend barriers for a risk process with stochastic return on investments. Insurance: Mathematics and Economics, 20:215-223.

[30] Paulsen, J. (2003). Optimal dividend payouts for diffusions with solvency constraints. Finance and Stochastics, 7(4):457-473.

[31] Paulsen, J. (2008). Optimal dividend payments and reinvestments of diffusion processes with both fixed and proportional costs. SIAM Journal on Control and Optimization, 47(5):2201-2226.

[32] Zhou, M. and Yuen, K. C. (2012). Optimal reinsurance and dividend for a diffusion model with capital injection: Variance premium principle. Economic Modelling, 29(2):198-207.

[33] Schmidli, H. (2008). Stochastic Control in Insurance. Springer-Verlag, London.

[34] Zhou, X. (2005). On a classical risk model with a constant dividend barrier. North American Actuarial Journal, 9:1-14.

School of Sciences, Hebei University of Technology, Tianjin 300401, P.R. China

E-mail address: liangxiaoqing115@hotmail.com

Faculty of Pure and Applied Mathematics, WrocŁaW University of SCience and TeChNOlogy, Wyb. WyspiańSKiego 27, 50-370 WrocŁaW, POLAND

E-mail address: zbigniew.palmowski@gmail.com 